Cohort study

\section{Study results suggest a positive effect of light-to-moderate physical activity on disability among those with or at risk of knee arthritis}

\subsection{6/ebmed-2014-110041}

\section{Maura D Iversen}

Department of Physical Therapy, Movement and Rehabilitation Sciences, Northeastern University; Brigham \& Women's Hospital, Boston, Massachusetts, USA

Correspondence to: Professor Maura D Iversen, Department of Physical Therapy, Movement and Rehabilitation Sciences, Northeastern University, 360 Huntington Avenue, Boston, MA 02115, USA; m.iversen@neu.edu

Commentary on: Dunlop DD, Song J, Semanik PA, et al. Relation of physical activity time to incident disability in community dwelling adults with or at risk of knee arthritis: prospective cohort study. BMJ 2014;348:g2472.

\section{Context}

Knee osteoarthritis (KOA) is a leading cause of disability worldwide and its prevalence increases with age. ${ }^{1}$ Annual costs due to disability in the USA reached nearly $\$ 357$ billion in $2010 .^{2}$ Data indicate that engagement in regular physical activity leads to improved physical function, endurance and mood state, and has the potential to reduce disability. ${ }^{3}$ This prospective cohort study examines the risk of incident disability and progression of disability in community-dwelling adults with or at risk for KOA.

\section{Methods}

This multisite study included 1680 community-dwelling adults aged 49 and older, either affected by or with established risk factors for KOA, to examine onset of disability. To determine progression of disability, a second cohort of 1814 adults without severe disability were gathered. All participants were enrolled in the Osteoarthritis Initiative. ${ }^{4}$ Individuals in the disability-onset cohort were classified as disability free in instrumental or basic activities of daily living at baseline.

The specific issues addressed in this study were: (1) whether moderate-to-vigorous physical activity is related to reduced risk of disability and (2) whether light physical activity levels are related to incident disability and progression of disability, independent of moderate-tovigorous levels of physical activity and other self-reported comorbidities.

Physical activity (minutes of light or moderate-to-vigorous intensity per day) was measured over seven consecutive days at baseline using accelerometers. A patient log was used to ascertain physical activities performed in water during the measurement week. Disability limitations were assessed using instrumental and basic activities of daily living at baseline and at 2 years. The primary outcome was incident disability. The secondary outcome was progression of disability, defined by increased level of disability at 2 years compared to baseline. Outcomes were reported as HRs and CIs. Sensitivity analyses were used to test assumptions.

\section{Findings}

Engagement in light intensity activities was inversely associated with development of disability. The highest frequency of incident disability occurred among the cohort with the least light-intensity and moderate-intensity physical activity, after adjusting for age. Both stratified and multivariate analyses indicated time spent in light physical activity was significantly associated with a reduced risk for developing disability. Data indicate that increasing quartiles of light physical activity corresponded with decreasing HRs for incident disability $(H R=1.00,0.62,0.51$ and $0.67 ; p=0.007)$. Depression and lower extremity pain were also significantly associated with incident disability. Greater moderate-to-vigorous physical activity participation was significantly associated with decreased risk of incident disability ( $\mathrm{HR}=1.00,0.57,0.63$ and $0.38 ; \mathrm{p}=0.005$ ). The impact of light physical activity participation appeared to be independent of moderate-to-vigorous physical activity engagement. With respect to disability progression, among adults determined to be without severe disability at baseline, both age-adjusted and multivariate models demonstrated a graded inverse relationship between light physical activity and disability progression ( $\mathrm{HR}=1.00$, $0.61,0.54$ and $0.60 ; p=0.018$ ). Similar trends were found for engagement in moderate-to-vigorous physical activity. Sensitivity analyses confirmed this significant inverse relationship between levels of light physical activity and disability onset and progression.

\section{Commentary}

This study addresses an important public health issue, specifically the impact of physical activity participation on disability onset and progression. As the authors note, there is insufficient data from randomised controlled trials to ascertain whether a dose relationship exists between engagement in physical activity and disability onset and progression. Using a prospective approach in a large, well-defined and diverse cohort of patients with KOA or risk for KOA provides data to suggest that light physical activity may independently be associated with disability onset and progression. Whether such physical activity engagement leads to reduced costs associated with KOA is yet to be determined.

Some assumptions made in modelling may attenuate the study's results. For example, measuring a single week of physical activity may not cover enough time to represent the physical activity profiles of adults at 2-year follow-up. Definitions of light physical activity using accelerometry data vary and may influence results. Changes in comorbidities over time or misclassification of baseline comorbidities due to measurement issues may also contribute to the progression and onset of disability, irrespective of KOA and its associated risk factors.

As the authors state, these data cannot confirm cause and effect. However, the consistent trends across analyses are promising. Despite the limitations of this study, the data suggest the need to examine, in randomised controlled trials, the influence of light physical activity on disability onset and progression among adults with KOA or at risk for KOA. Such data would be instrumental in designing public health programmes to address physical activity and inform rehabilitation interventions designed to enhance functional outcomes of patients with KOA.

Competing interests None.

Provenance and peer review Commissioned; internally peer reviewed.

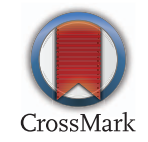

\section{References}

1. Dillon CF, Rasch EK, Gu Q, et al. Prevalence of knee osteoarthritis in the United States: arthritis data from the third National Health and Nutrition Examination Survey 1991-94. J Rheumatol 2006;33:2271-9.

2. Brault MW. Americans with disabilities: 2010. US Bureau of the Census, US Department of Commerce, 2012:1-23.

3. Chronic Osteoarthritis Management Initiative. COAMI Management Conference 2013: executive summary. United States Bone and Joint Initiative.

4. Osteoarthritis Initiative. Data news. 2013. http://www.oai.ucsf.edu/datarelease/ 